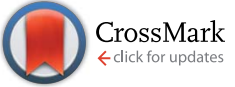

Cite this: RSC Adv., 2017, 7, 31

Received 21st October 2016 Accepted 7th December 2016

DOI: 10.1039/c6ra25636h

www.rsc.org/advances

\title{
A free-standing porous silicon-type gel sponge with superhydrophobicity and oleophobicity $\dagger$
}

\author{
Xia Zhang, *ab Wenzhong Zhu ${ }^{a}$ and Ivan P. Parkin*b \\ Porous and spongy superhydrophobic silicon-type gels monoliths have been fabricated by a facile method. \\ The gel can be turned oleophobic after modification with fluorosilane. The as-prepared free-standing \\ silicon-type gels demonstrate superhydrophobicity and oleophobicity not only on the upper surface but \\ also throughout the whole monolith. Importantly, the as-prepared amphiphobic silicon-type gels have \\ remarkable chemical stability even in corrosive solutions over a wide range of $\mathrm{pH}$ conditions. Moreover, \\ the silicon-type gel monoliths also have stable amphiphobic properties even after UV irradiation and \\ long-term storage in air.
}

\section{Introduction}

Recently, amphiphobic surfaces have drawn much attention because of their applications in many areas, such as selfcleaning, ${ }^{1-3}$ drug-reduction, ${ }^{4}$ corrosion resistance, ${ }^{5}$ antifouling, ${ }^{6}$ and anti-icing. ${ }^{7}$ However, the preparation of such amphiphobic surfaces presents a greater challenge, since oil liquids such as cooking oils have a lower surface tension compared to that for water. ${ }^{8}$ Furthermore, most amphiphobic surfaces have poor durability. ${ }^{9-12}$ Chemical oxidation from exposure to air, a particular chemical environment, strong light, or physical rubbing could cause the surfaces to permanently lose their water or oil repellency, which restricts their prospects in industrial applications. ${ }^{13}$ Recently, Barthwal et $a{ }^{14}{ }^{14}$ generated a mechanically robust superamphiphobic surface on an aluminum plate by employing a simple etching process in an acidic solution followed by anodization and fluorination. Similarly, Lee et $a{ }^{15}{ }^{15}$ fabricated copper oxide nanowires on copper substrates by an oxidation-reduction process and checked the mechanical stability of the surfaces. However, these studies showed that the fragile nature of the nanostructures were damaged or worn off by contact at higher loads. It is imperative to improve the durability of amphiphobic surfaces for practical applications. ${ }^{\mathbf{1 0 , 1 6}}$ The advantage for elastic materials of improving the abrasion resistance of amphiphobic surfaces is caused by their elasticity, since the

${ }^{a}$ National \& Local Joint Engineering Research Center for Applied Technology of Hybrid Nanomaterials, Henan University, Kaifeng 475004, P. R. China. E-mail: xia.zhang@ ucl.ac.uk

${ }^{b}$ Department of Chemistry, University College London, 20 Gordon Street, London, WC1H 0AJ, UK. E-mail: i.p.parkin@ucl.ac.uk

$\dagger$ Electronic supplementary information (ESI) available: (1) Video showing the separation of water and organic liquids by superhydrophobic silicon-type gels; (2) video showing the floating behavior of the silicon-type gels after being pressed under organic liquids. See DOI: 10.1039/c6ra25636h surface can spring back after compression or scratch and avoid the damage of the surface micro- and nanostructures. ${ }^{\mathbf{1 7 - 2 1}}$ Moreover, if the amphiphobicity is sustained throughout the whole monolith material volume, it can foster formation of fresh re-entrant surface with amphiphobic property after removed of the uppermost damaged layer. ${ }^{22,23}$ In our previous work, we fabricated superhydrophobic elastic silicone rubber with excellent mechanical durability and showed the superhydrophobicity when exposed to harsh environments such as strong corrosion, extremely low temperature, various external damages, especially high mechanical stress. ${ }^{24}$ In this work, we present a template-free procedure for the fabrication of marshmallow-like silicon monoliths with superhydrophobic and oleophobic properties. No template and stabilizer were required during this process, which is of low environmental impact, low cost, and simple to make. The obtained silicon-type gels sustain their amphiphobicity not only on the outside surface but also throughout the whole volume, thus when the outmost layer is removed, the renewed layer retain the superhydrophobic and oleophobic property making the amphiphobicity permanent. In addition, the silicon-type gels own soft and porous structures are surprisingly very stable to strong acid or alkali conditions.

\section{Experimental}

2.1. Fabrication of free-standing superhydrophobic silicontype gels

First, $0.80 \mathrm{~g}$ of hexadecyltrimethylammonium bromide, $5.0 \mathrm{~g}$ of urea, and $15 \mathrm{~mL}$ of $5 \mathrm{mM}$ aqueous acetic acid were mixed. Then $3.0 \mathrm{~g}$ of methyltrimethoxysilane and $2.0 \mathrm{~g}$ of dimethyldimethoxysilane were added at the same time under vigorous stirring at ambient temperature until the solutions were homogeneous. The obtained sol was transferred into a tightly sealed container, which was placed in a forced convection oven at $80^{\circ} \mathrm{C}$ for about 
$10 \mathrm{~h}$ for complete gelation and aging. Note that stirring should not be done during the whole aging process. After that, the obtained silicon-type gels monoliths were carefully washed with isopropyl alcohol by soaking/squeezing for several times to remove the residual surfactant and other chemicals. The washed samples were dried under ambient conditions to obtain superhydrophobic and superoleophilic gels.

\subsection{Fabrication of oleophobic silicon-type gels}

The obtain silicon-type gels were immersed in a $0.5 \% n$-hexane solution of 2-(perfluorodec-1-yl)ethyltrichlorosilane $\left[\mathrm{CF}_{3}(-\right.$ $\left.\mathrm{CF}_{2}\right)_{9}\left(\mathrm{CH}_{2}\right)_{2} \mathrm{SiCl}_{3}$, FDTS] for about $24 \mathrm{~h}$ at room temperature. Then the samples were taken from the FDTS solution and dried in an oven at $70{ }^{\circ} \mathrm{C}$ for $3 \mathrm{~h}$.

\subsection{Characterization}

The surface morphology of the sample was examined by field emission scanning electron microscopy (FESEM, JSM-6701F). The chemical composition of the as-prepared surface was investigated using X-ray photoelectron spectroscopy (XPS), which was conducted on a PHI-5702 electron spectrometer using $\mathrm{Mg} \mathrm{K} \alpha$ line as an excitation source with reference of $\mathrm{C} 1 \mathrm{~s}$ at $284.8 \mathrm{eV}$. Apparent CA and SA were measured with 5 droplets of water and various organic liquids using a KRüSS DSA 100 apparatus. The average $\mathrm{CA}$ and SA values were obtained by measuring the same sample in at least five different positions, and images were captured with a digital camera (Sony Inc., Japan).

\section{Results and discussion}

Hydrolytic condensation of organosilanes through a one-pot sol-gel reaction can form a silicon-oxygen core frame-work and finally leads to a free-standing bulk silicon-type gels through the aging process. ${ }^{25}$ As shown in Fig. 1a-c, the freestanding gels can be prepared in diverse shapes and water droplets on the surface or cut inner surface of the bulk sample with a water contact angle about $158 \pm 1^{\circ}$ and a low sliding angle $2 \pm 0.5^{\circ}$. Once an oil droplet is dropped on the surface of the superhydrophobic silicon-type gels before functionalization with fluorosilane, the oil can immediately spread with the contact angle about $0^{\circ}$, which demonstrates the superoleophilic property. The superhydrophobic and superoleophilic silicontype gels monoliths can provide an extremely large volume for the entrance and storage of liquids, and can be used as an absorption media for the quick removal of unwanted organic liquids. Herein, we report the outstanding capability of these bulk materials for absorbing organic liquids and investigate the oil-absorption capacity. According to previous reports, ${ }^{26}$ the oilabsorption capacity of the sponge was calculated as follows:

$$
\text { Weight gain }(\%)=\left(M_{\text {after }}-M_{\text {before }}\right) / M_{\text {before }} \times 100 \%
$$

where $M_{\text {before }}$ was the weight of the superhydrophobic silicontype gels before adsorption (mg) and $M_{\text {after }}$ was the weight of the superhydrophobic silicon-type gels after adsorption (mg). As shown in Fig. 1d and Video S1, $\uparrow$ once a piece of the superhydrophobic sponge was placed on the surface of the oil-water mixtures, the oils were quickly absorbed by the sponge in a few
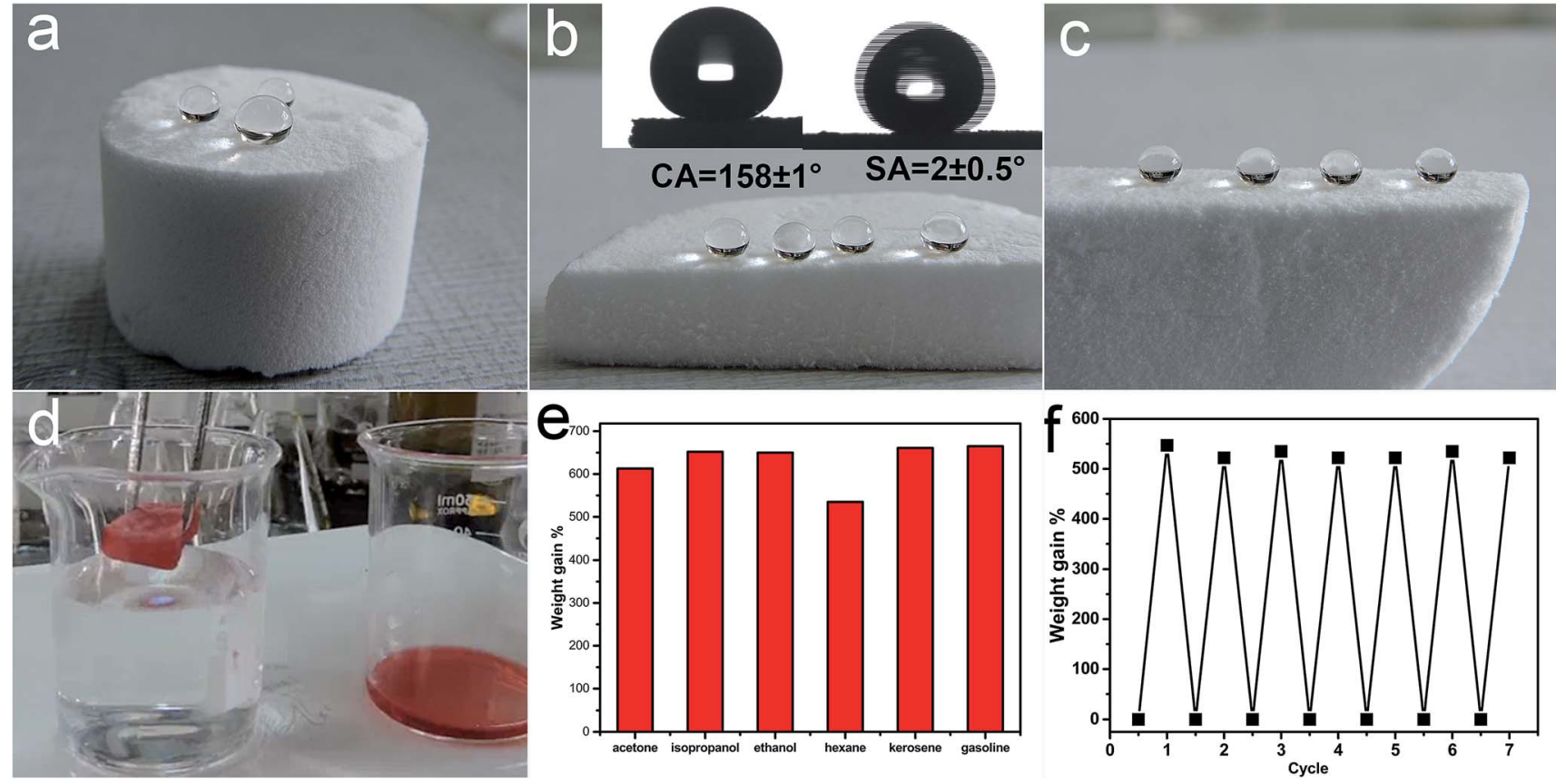

Fig. 1 Optical images of the water droplets $(4-8 \mathrm{mg})$ placed on the as-prepared bulk (a and b) or cut (c) silicon-type gels surface with a contact angle of $159 \pm 1^{\circ}$ and sliding angle of $1 \pm 0.5^{\circ}$. (d) Photograph of the absorption process of chloroform by using a superhydrophobic silicon sponge. (e) Absorption efficiency of the superhydrophobic silicon-type gels for various organic liquids. (f) Hexane absorption/drying cycle. 


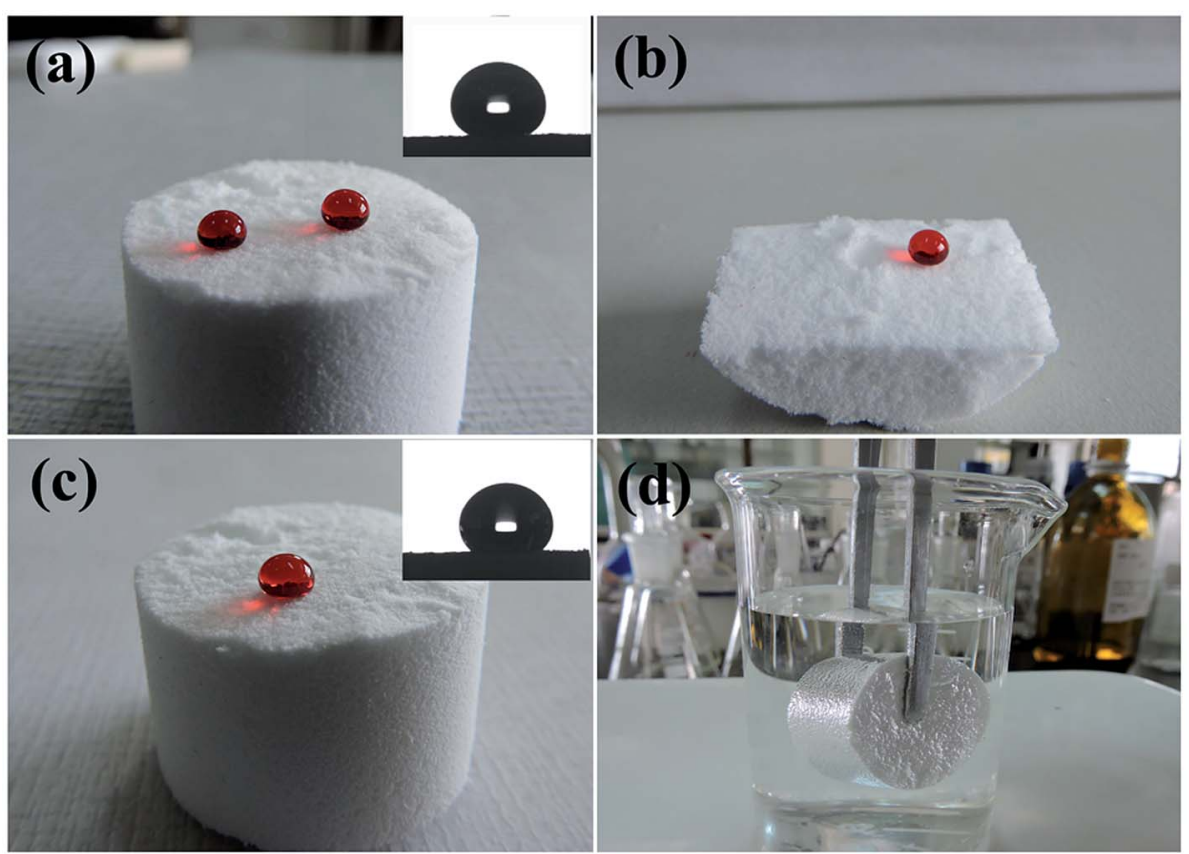

Fig. 2 Photograph of DMSO droplet on (a) the bulk silicon-type gels surface and (b) the cut inner surface. (c) Tetradecane droplet on the bulk silicon-type gels surface. (d) Mirror-like phenomenon can be observed on the bulk material submerged in water (DMSO and tetradecane are stained with oil red $\mathrm{O}$ ).

seconds. The superhydrophobic sponge exhibits different weight gains for the 6 kinds of oils/organic solvents and the changes in absorption capacity could be as a result of the difference in density of the oils/organic solvents (Fig. 1e). After being rinsed thoroughly with alcohol and dried in an oven at $70{ }^{\circ} \mathrm{C}$ for half an hour, the sample recovered its original shape and could be reused for oil/water separation for many cycles. As illustrated in Fig. 1f, only minor changes in absorption capability of the superhydrophobic sponges could be observed after absorption/drying cycles. This result is very important for the silicon monolith as a recyclable absorbent material in oils/ organic solvents cleanup applications. However, after the modification with fluorosilane (FDTS), the bulk sponge demonstrates both superhydrophobic and oleophobic
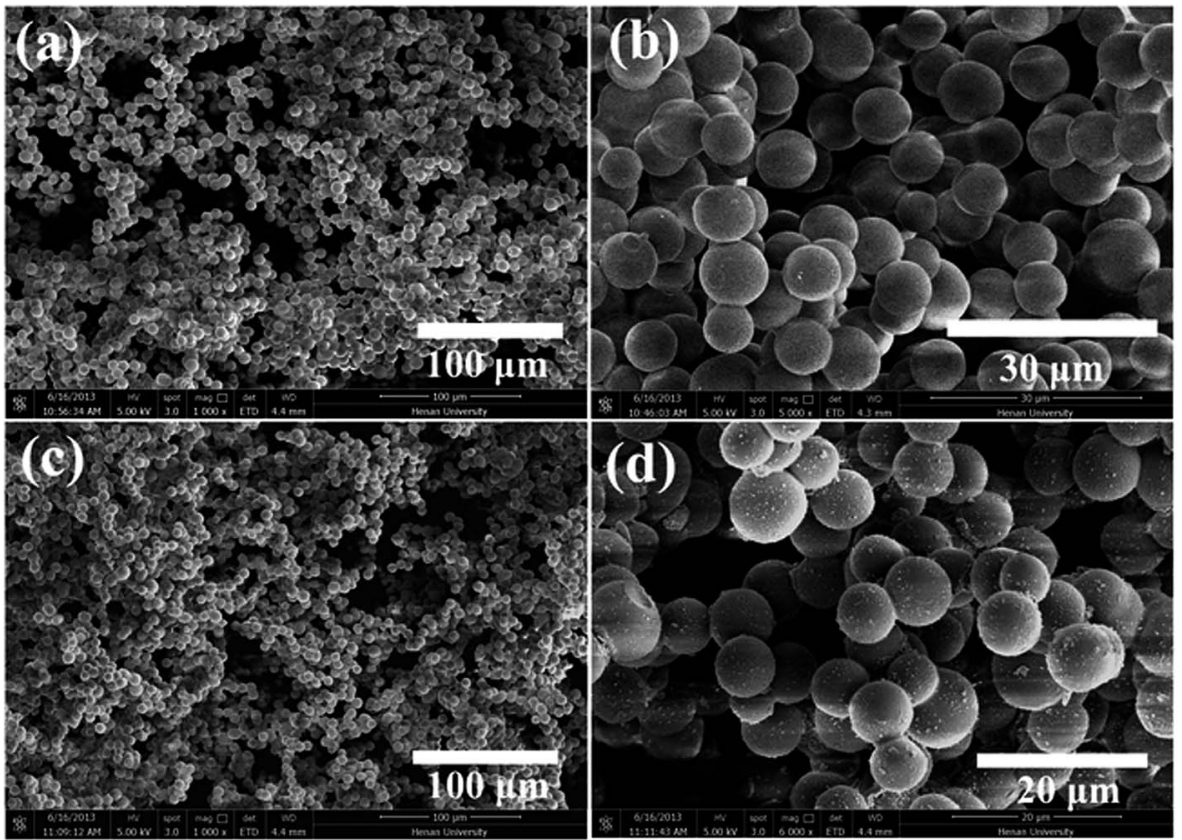

Fig. 3 SEM images of silicon-type gels before ( $a$ and $b$ ) and after ( $c$ and $d$ ) functionalization with the FDTS. 
properties extending throughout its bulk. As shown in Fig. 2a and c, dimethylsulfoxide (DMSO) and tetradecane droplets exhibit typical spherical shapes with CA value about $152 \pm 1^{\circ}$ and $146 \pm 1^{\circ}$, respectively. Note that the cut bulk silicon-type gels also retain oleophobicity, and that the DMSO droplet can keep its typical spherical shape with CA value about that $150^{\circ} \pm$ $1^{\circ}$ (Fig. 2b). Upon immersion in water, the bulk material surface acts like a sliver mirror when viewed at a glancing angle (Fig. 2d), due to the total reflectance of light at the air layer trapped on the surface. This trapped air can effectively prevent a wetting on the fabric surface underwater. ${ }^{27}$ Interestingly, when the amphiphobic sponge is immersed even in oil by an external force, it would instantaneously float up after the force is released, and keeps completely dry. The liquid repellency of the bulk sponge is highlighted in Movie S2 (ESI $\dagger$ ). Thus, the high liquid repellency of the amphiphobicity can prevent the sponge from liquid pollution.

SEM was used to clarify the morphology of the spongy silicon-type gels before and after functionalization with the FDTS. As illustrated in Fig. 3a and b, the superhydrophobic and superoleophilic silicon-type gels surfaces contain micrometer spherical particles, and each particle is tightly bound together at the neck, forming a rough and porous structure. Compared

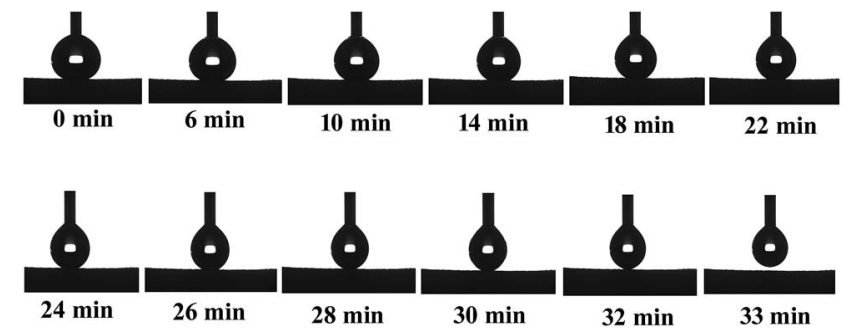

Fig. 5 Evolution of water droplet on amphiphobic silicon-type gels surface.

with the sponge before modification, the amphiphobic sponge has almost the same morphology (Fig. 3c and d). The variation of chemical composition is the main cause of the different wettability. XPS measurement was used to investigate the surface chemical composition of the sample. For the superhydrophobic and superoleophilic sponge, only peaks corresponding to $\mathrm{Si}, \mathrm{C}$ and $\mathrm{O}$ were observed (Fig. 4a). After the deposition process, new peaks appeared as shown in Fig. $4 \mathrm{~b}$, which were attributed to $F_{1 s}$, and $F_{K L L}$. The $C_{1 s}$ peak can be deconvoluted into four peaks at 284.8, 286.3, 292.4, and $293.3 \mathrm{eV}$, which are assigned to $\mathrm{C}-\mathrm{C} / \mathrm{C}-\mathrm{H}, \mathrm{CH}_{2}-\mathrm{CF}_{2},-\mathrm{CF}_{2}-$ and (a)

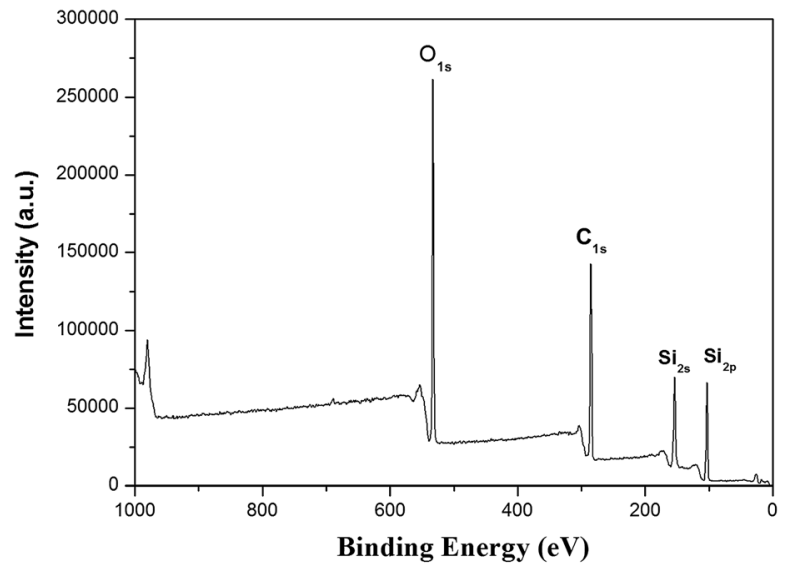

(c)

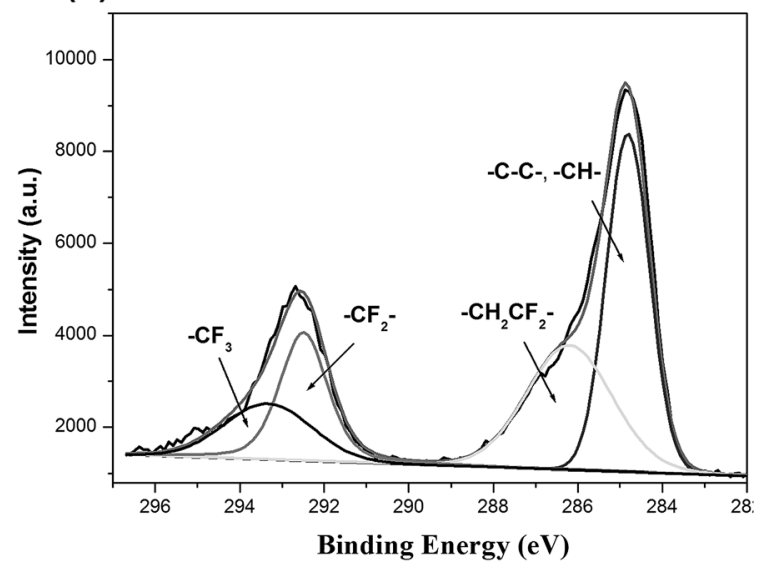

(b)

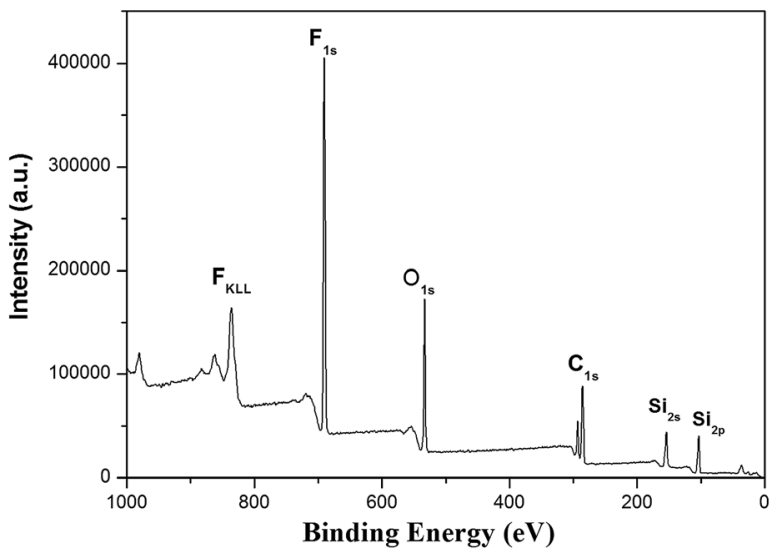

(d)

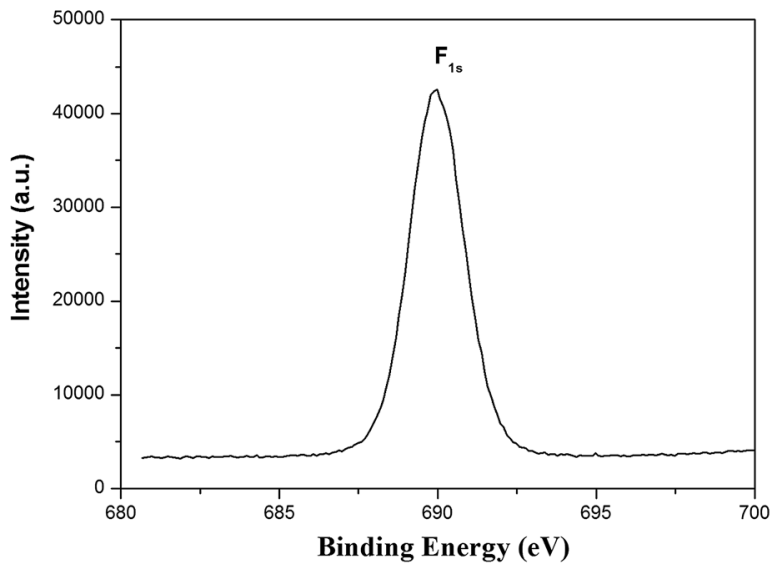

Fig. 4 XPS spectra of silicon-type gels before (a) and after (b) functionalization with the FDTS; (c and d), C and F element profile from XPS of gels functionalization with FDTS. 
(a)

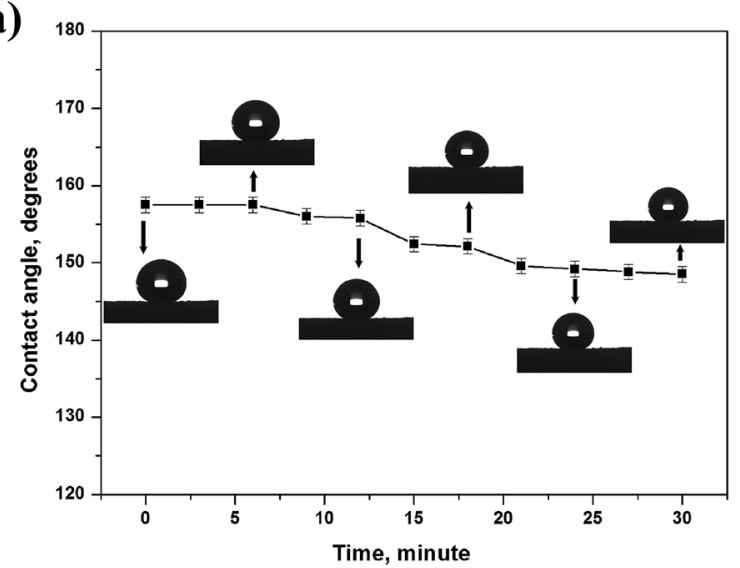

(b)

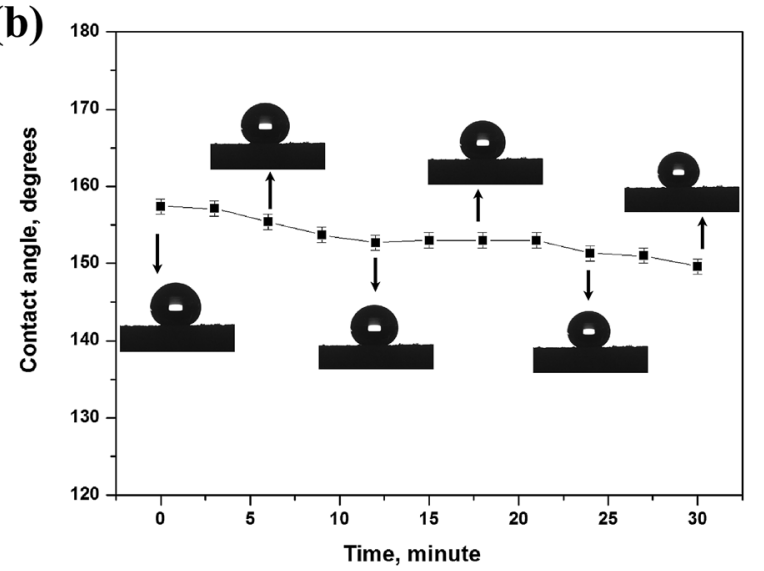

Fig. 6 Contact angle on amphiphobic silicon-type gels in the condition of continuous contact with the corrosive water droplet. (a) Acidic (pH = 1) and (b) alkali ( $\mathrm{pH}=14)$.

$-\mathrm{CF}_{3}$ groups, respectively (Fig. 4c). The peak located at about $690.5 \mathrm{eV}$ is ascribed to $\mathrm{F}_{1 \mathrm{~s}}$ in the PDTS (Fig. $4 \mathrm{~d}$ ). It is well-known that the $-\mathrm{CF}_{3}$ group has the lowest surface energy (the theoretical surface energy is $6 \mathrm{mN} \mathrm{m}^{-1}$ ), and that of the $-\mathrm{CF}_{2}$ is the next lowest. ${ }^{28}$ The high surface concentration of $-\mathrm{CF}_{2}$ and $-\mathrm{CF}_{3}$ groups results in a surface with the low surface energy needed to achieve amphiphobicity.

The evolution of the contact angle in continuous contact with aqueous media is of primary importance for revealing the possible applications of such silicon-type gels surfaces. Herein, the durability of superhydrophobic properties under continuous contact with water was investigated. Fig. 5 shows the contact angle evolution of an amphiphobic silicon-type gels after continuous contact with a water droplet. It is clear that the water droplet keeps its spherical shape with CA value about $158^{\circ}$. However, with increased time, the droplet size decreases because of evaporation. After staying on the sponge surface for about $30 \mathrm{~min}$, the water droplet detaches from the surface spontaneously and sticks to the hydrophilic needle, which indicates that the superhydrophobic property is stable with low adhesion of water of the surface.

As far as the acidic $(\mathrm{pH}=1)$ and alkali $(\mathrm{pH}=14)$ liquids are concerned, the sponge can be not as resistant as it is towards pure water but still demonstrates a significant degree of stability. It may be concluded that increasing the contact time with corrosive liquid droplets facilitates the degradation of superhydrophobic properties. It is clear that the sponge keeps its superhydrophobic property for about only $20 \mathrm{~min}$ after dropping a strong acidic droplet (Fig. 6a). Meanwhile, a strong alkali droplet can keep the CA value larger than $150^{\circ}$ for $30 \mathrm{~min}$ (Fig. 6b). These results indicate that the amphiphobic sponge has a certain sustainable stability over a wide $\mathrm{pH}$ range, which is very important for the application in a corrosive environment.

\section{Conclusions}

In conclusion, we have demonstrated a facile method for the fabrication of flexible superhydrophobic and oleophobic silicon-type gel surface. The evolution of water contact angle in time under the condition of continuous contact with corrosive droplets shows that the amphiphobic silicon-type gels surface has a high degree of chemical stability. The elastic silicon-type gel surface also keeps its stable amphiphobic property under UV irradiation and after long-term storage in air. This study is expected to provide new opportunities for the production of largescale superhydrophobic and oleophobic surfaces for many industrial applications such as self-cleaning, corrosion resistance and antifouling.

\section{Acknowledgements}

This work was supported by the National Nature Science Foundation of China (Grant No. 21403055), Joint Talent Cultivation Funds of NSFC-HN (Grant No. U1304529).

\section{References}

1 L. V. Li, D. Breedveld and W. Hess, ACS Appl. Mater. Interfaces, 2013, 5, 5381-5386.

2 J. Xi, L. Feng and L. A. Jiang, Appl. Phys. Lett., 2008, 92, 053102.

3 T. L. Sun, L. Feng, X. F. Gao and L. Jiang, Acc. Chem. Res., 2005, 38, 644-652.

4 C. Lee and C. J. Kim, Phys. Rev. Lett., 2011, 106, 014502.

5 H. Q. Liu, S. Szunerits, W. G. Xu and R. Boukherroub, ACS Appl. Mater. Interfaces, 2009, 1, 1150-1153.

6 J. Genzer and K. Efimenko, Biofouling, 2006, 22, 339-360.

7 M. Ma and R. M. Hill, Curr. Opin. Colloid Interface Sci., 2006, 11, 193-202.

8 G. W. Zhang, S. D. Lin, I. Wyman, H. L. Zou, J. W. Hu, G. J. Liu, J. D. Wang, F. Li, F. Liu and M. L. Hu, ACS Appl. Mater. Interfaces, 2013, 5, 13466-13477.

9 H. Zhou, H. X. Wang, H. T. Niu, A. Gestos and T. Lin, Adv. Funct. Mater., 2013, 23, 1664-1670.

10 T. Verho, C. Bower, P. Andrew, S. Franssila, O. Ikkala and R. H. A. Ras, Adv. Mater., 2011, 23, 673-678.

11 P. Roach, N. J. Shirtcliffe and M. I. Newton, Soft Matter, 2008, 4, 224-240. 
12 X. Zhang, Y. G. Guo, H. Z. Chen, W. Z. Zhu and P. Y. Zhang, J. Mater. Chem. A, 2014, 2, 9002-9006.

13 H. X. Wang, Y. H. Xue, J. Ding, L. F. Feng, X. G. Wang and T. Lin, Angew. Chem., Int. Ed., 2011, 50, 11433-11436.

14 S. Barthwal, Y. S. Kim and S. H. Lim, Langmuir, 2013, 29, 11966-11974.

15 S. M. Lee, K. S. Kim, E. Pippel, S. Kim, J. H. Kim and H. J. Lee, J. Phys. Chem. C, 2012, 116, 2781-2790.

16 X. Li, L. L. Kendrick, H. Heusser, J. C. Webb, B. J. Sparks, J. T. Goetz, W. Guo, M. Stafford, M. D. Blanton, S. Nazarenko and D. L. Patton, ACS Appl. Mater. Interfaces, 2014, 6, 10763-10774.

17 J. J. Tang, Y. Q. Xu, W. Zhang, X. Q. Yuan, Y. Liu and C. H. Su, Physics, 2013, 52, 1558-1566.

18 G. Hayase, K. Kanamori, M. Fukuchi, H. Kaji and K. Nakanishi, Angew. Chem., Int. Ed., 2013, 52, 1986-1989.

19 G. Hayase, K. Kanamori and K. Nakanishi, J. Mater. Chem., 2011, 21, 17077-17079.

20 G. Hayase, K. Kugimiya, M. Ogawa, Y. Kodera, K. Kanamori and K. Nakanishi, J. Mater. Chem. A, 2014, 2, 6525-6531.
21 K. Kanamori, Y. Kodera, G. Hayase, K. Nakanishi and T. Hanada, J. Colloid Interface Sci., 2011, 357, 336-344.

22 L. X. Li and J. P. Zhang, Adv. Mater. Interfaces, 2016, 3, 1600517-1600524.

23 G. Hayase, K. Kanamori, G. Hasegawa, A. Maeno, H. Kaji and K. Nakanishi, Angew. Chem., 2013, 125, 10988-10991.

24 X. Zhang, W. Z. Zhu, G. J. He, P. Y. Zhang, Z. J. Zhang and I. P. Parkin, J. Mater. Chem. A, 2016, 4, 14180-14186.

25 J. M. Mabry, A. Vij, S. T. Iacono and B. D. Viers, Angew. Chem., Int. Ed., 2008, 47, 4137-4140.

26 B. Wang, J. Li, G. Y. Wang, W. X. Liang, Y. B. Zhang, L. Shi, Z. G. Guo and W. M. Liu, ACS Appl. Mater. Interfaces, 2013, 5, 1827-1839.

27 I. A. Larmour, S. E. J. Bell and G. C. Saunders, Angew. Chem., Int. Ed., 2007, 46, 1710-1712.

28 K. Ellinas, S. P. Pujari, D. A. Dragatogiannis, C. A. Charitidis, A. Tserepi, H. Zuilhof and E. Gogolides, ACS Appl. Mater. Interfaces, 2014, 6, 6510-6524. 